\title{
IMPROVED MULTIPLE TRAJECTORIES ROTATOR DESIGN FOR TRANSFORMS
}

\author{
Pratik Trivedi \\ Institute of Technology, Nirma University, Ahmedabad, India \\ Zaveri Tanish \\ Institute of Technology, Nirma University, Ahmedabad, India
}

\begin{abstract}
Any base from one domain to other domain can be converted through some transformation. Hence transforms play a major role in any signal processing applications. It will involve some basis function to convert from one domain from the other. Initially to calculate the coefficients of any transformation the techniques like look up table based, CORDIC based are well known. The paper talks about the various techniques and their real time implementations results. Results shows improvement in calculations if quadrant mapping and the orders of the transforms are changed.
\end{abstract}

Keywords: CORDIC, DCT, FFT, Signal Processing

Cite this Article: Pratik Trivedi and Zaveri Tanish, Improved Multiple Trajectories Rotator Design for Transforms, International Journal of Advanced Research in Engineering and Technology (IJARET), 10 (6), 2019, pp 140-149.

http://iaeme.com/Home/issue/IJARET?Volume $=10 \&$ Issue $=6$

\section{INTRODUCTION}

Fourier transforms convert a signal to and from the frequency domain, by breaking a signal down into its frequency components. The fast Fourier transform (FFT) reduces the number of calculations of the DFT by dividing the initial function into repeated sub-functions and continues this process until the sub-function is no longer divisible.

Hence, reducing the computational complexity while using transforms in real-life applications becomes necessary. For the calculations, to achieve maximum performance, we need to optimize the hardware (i.e. the number of shifters, adders and multipliers). This will lead to reduction in the time complexity and the size of the architecture implementation, which in turn reduces the cost and power. This modified algorithm has advantages of reduced hardware and improved latency as the speed of the architecture is enhanced.

\section{MULTIPLICATION BY TWO INTEGERS USING THE MINIMUM NUMBER OF ADDERS [1]}

This concept talks about the minimum adder graph algorithm which designs shifters and adder circuits that aid multiplication by integers using the minimum number of adders. It 
considers the circuit as a graph made up of two input adders. It performs an exhaustive search of all possible graph topologies and produces two tables out of which one contains the number of adders required to produce the circuit and other contains the partial products of the adders. Further this paper talks about reusing the repeating products which come up while multiplying two numbers. So, if an adder block has the same two inputs as before it doesn't need to calculate that again. Also, it takes care of cases in which one or two inputs of the adders are powers of 2 . If that is the case, no extra adders are required as that can be achieved with shifters alone. Thus, the algorithm proposed by the paper achieves the fewest adders required in a circuit.

\section{CORDIC [5]}

CORDIC stands for the Co-ordinate Rotation in Digital Computer. The CORDIC algorithm works on transforming a vector by a given angle and getting the resultant vector. For that we specified a number of iterations post which the algorithm terminates. The algorithm works by making micro-rotations of the input vector in the direction of the difference between the destination angle and the angle at the current iteration. CORDIC reduces the memory taken up by a look up table (LUT). Another approach which can be thought of is the series expansion of sines and cosines in the rotation matrix but that will increase the number of adders and multipliers required. Hence CORDIC is used. Here it is assumed that the tangent of an angle is the negative power of 2 . Using this angle $i$ continuously iterate till the number of iterations specified and move in the direction of the difference between the current angle and the destination angle.

$$
\begin{aligned}
& {\left[\begin{array}{l}
X i+1 \\
Y i+1
\end{array}\right]=\left[\begin{array}{l}
x i \\
y i
\end{array}\right]\left[\begin{array}{cc}
\cos \theta i & -\sin \theta i \\
\sin \theta i & \cos \theta i
\end{array}\right]} \\
& {\left[\begin{array}{l}
X i+1 \\
Y i+1
\end{array}\right]=\left[\begin{array}{l}
x i \\
y i
\end{array}\right] \cos \theta i\left[\begin{array}{cc}
1 & -\tan \theta i \\
\tan \theta i & 1
\end{array}\right]}
\end{aligned}
$$

Where $\tan \theta i=2^{-i}$

\section{UNIFIED CORDIC [3] [4]}

CORDIC algorithms then can be computed with help of linear, circular, and hyperbolic trajectories. And depending upon their equation, trajectories of the vectors will be defined and generated by the successive iterations.

$$
\begin{aligned}
& X i+1=x i-m d i y i 2^{-i} \\
& Y i+1=y i+d i x i 2^{-i}
\end{aligned}
$$

Where $\mathrm{m}=0,1$ and -1 for linear circular and hyperbolic trajectories.

Out of these three categories, circular and hyperbolic are more prevalent and widely used. Circular CORDIC is mainly used for the computation of sine/cosine functions, waveform generation, implementation of digital filters, transform computation, matrix calculations etc., whereas, hyperbolic CORDIC is used for the computation of exponents and sinh/cosh functions, neural networks. CODIC has a wide range of applications and is also used to simplify other basic and important algorithms like Eigen-value estimations, QR decomposition, phase and frequency estimations, singular value decomposition, synchronization in digital receivers, graphics processing and robot manipulation, both rotation and vectoring-modes. The hardware implementation of these applications requires more than one CORDIC processor operating in different modes and trajectories, but we do not find any reconfigurable CORDIC. 
As the equations of the hyperbolic, circular, and linear CORDIC are different, therefore each will have a different architecture. The paper works on bringing all the different trajectories into a single combined architecture, thus the presents reconfigurable CORDIC (Co-ordinate Rotation Digital Computer) architectures which can be configured to operate either for circular or hyperbolic trajectories in rotation as well as vectoring-modes by manipulating equations in such a way that changing one value may change to either of the remaining two algorithms. We propose three reconfigurable CORDIC designs: a reconfigurable rotation mode CORDIC that operates either for circular or hyperbolic trajectory, a reconfigurable vectoring-mode CORDIC for circular and hyperbolic trajectories, and a generalized reconfigurable CORDIC that can operate in any of the modes for both circular as well as hyperbolic trajectories. The reconfigurable CORDIC can perform the computation of various trigonometric and exponential functions, logarithms, square-root, etc. of circular and hyperbolic CORDICs using either rotation-mode or vectoring mode of operation in one single circuit. The various applications include digital synchronizers, graphics processors, scientific calculators and many other applications. Because all the 3 architectures are implemented in one architecture and in a combined way, it saves a lot of area as well.

\section{ENHANCED CORDIC [2]}

In the traditional CORDIC algorithm there is a multiplier required at each iteration (due to the cos term also known as scale factor) of the algorithm which increases the hardware complexity. Hence enhanced CORDIC was designed whose accuracy will be less than traditional CORDIC due to the low order of the series expansion of sines and cosines but the hardware complexity is reduced as the need for a multiplier is eliminated. In enhanced CORDIC the series expansion of sines and cosines are taken up till the second order.

$$
\begin{aligned}
& \sin x=x-\frac{x}{3 !}^{3}+\frac{x}{5 !}^{5}-\frac{x}{7 !}^{7} \\
& \cos x=1-\frac{x}{2}^{2}+\frac{x}{4 !}^{4}-\frac{x}{6}^{6}
\end{aligned}
$$

This brings down the enhanced CORDIC equations as

$$
\begin{aligned}
& X i+1=x i\left(1-\frac{x}{2 !}^{2}+\frac{x^{4}}{4 !}-\frac{x^{6}}{6 !}+\cdots\right)-y i\left(\mathrm{x}-\frac{x}{3 !}^{3}+\frac{x}{5}^{5}-\frac{x}{7}^{7}\right) \\
& Y i+1=y i\left(1-\frac{x}{2 !}^{2}+\frac{x}{4 !}^{4}-\frac{x}{6 !}^{6}+\cdots\right)+x i\left(\mathrm{x}-\frac{x}{3 !}^{3}+\frac{x}{5 !}^{5}-\frac{x}{7 !}^{7}\right)
\end{aligned}
$$




\section{IMPLEMENTATION AND RESULTS}

\subsection{CORDIC}

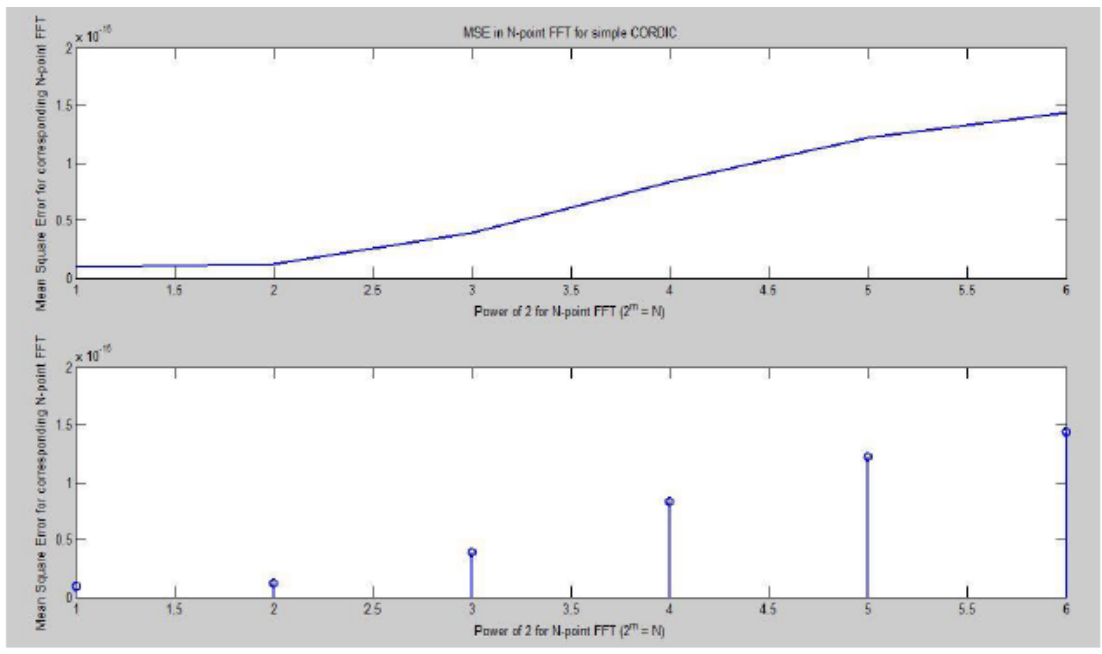

Figure 1 Simple CORDIC: FFT points till 64-point MSE result (i=55)

Table 1

MSE in Simple CORDIC for N-point FFT with varying ' $i$ '

\begin{tabular}{|c|c|c|c|c|c|c|}
\hline \multicolumn{7}{|c|}{ MSE in Simple CORDIC for $\mathrm{N}$-point FFT with varying ' $\mathrm{i}$ ' } \\
\hline \multirow{3}{*}{ \#iterations: 'i' } & \multirow{2}{*}{\multicolumn{6}{|c|}{ Mean Square Error }} \\
\hline & & & & & & \\
\hline & 2-point & 4-point & 8-point & 16-point & 32-point & 64-point \\
\hline 50 & 0.0209 & 0.0177 & 0.0165 & 0.3344 & 0.6269 & 0.2936 \\
\hline 53 & 0.0126 & 0.0137 & 0.0549 & 0.1096 & 0.1211 & 0.1095 \\
\hline 55 & 0.0098 & 0.0121 & 0.0392 & 0.0838 & 0.1219 & 0.1434 \\
\hline 57 & 0.0091 & 0.0117 & 0.0393 & 0.0917 & 0.1215 & 0.1362 \\
\hline 59 & 0.0093 & 0.0118 & 0.0392 & 0.0917 & 0.1215 & 0.1362 \\
\hline 100 & 0.0092 & 0.0117 & 0.0393 & 0.0917 & 0.1215 & 0.1362 \\
\hline 500 & 0.0092 & 0.0117 & 0.0393 & 0.0917 & 0.1215 & 0.1362 \\
\hline
\end{tabular}

From the above graph and table, we could analyze and conclude that ROC for circular trajectory $(\mathrm{m}=1)$ was 90 degress. If we try for an angle greater than this, the error increased. Hence, here we further implemented qudrant mapping in this where we could simply map the value of the first quadrant in the other three (with change in signs). This reduced complexity and error. 


\subsection{Enhanced CORDIC Results ( $2^{\text {nd }}$ order)}

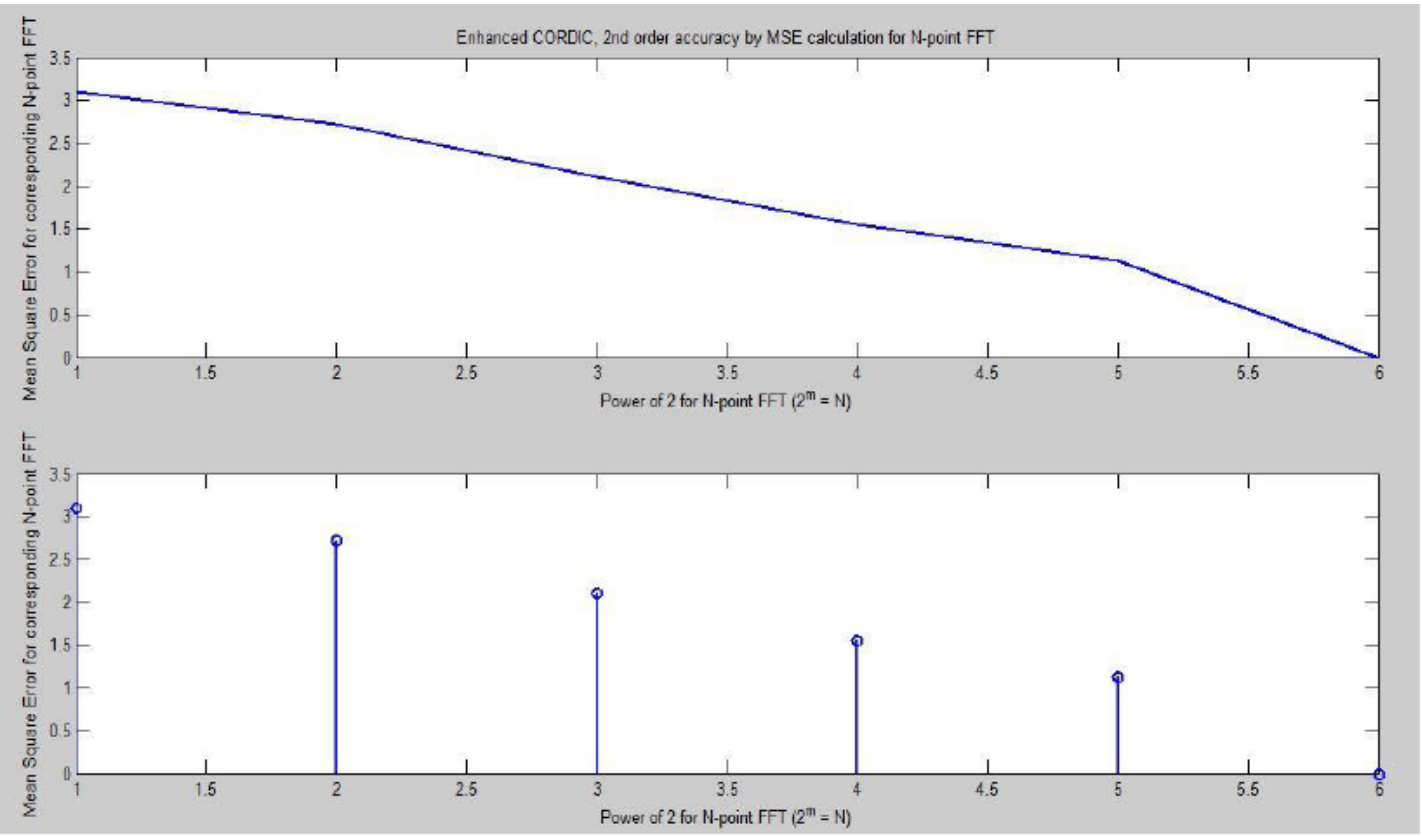

Figure 2 Enhanced CORDIC: FFT point till 64-point MSE result ( $2^{\text {nd }}$ order accuracy)

\subsection{Extended Enhanced CORDIC Algorithm (5 ${ }^{\text {th }}$ order $)$,}
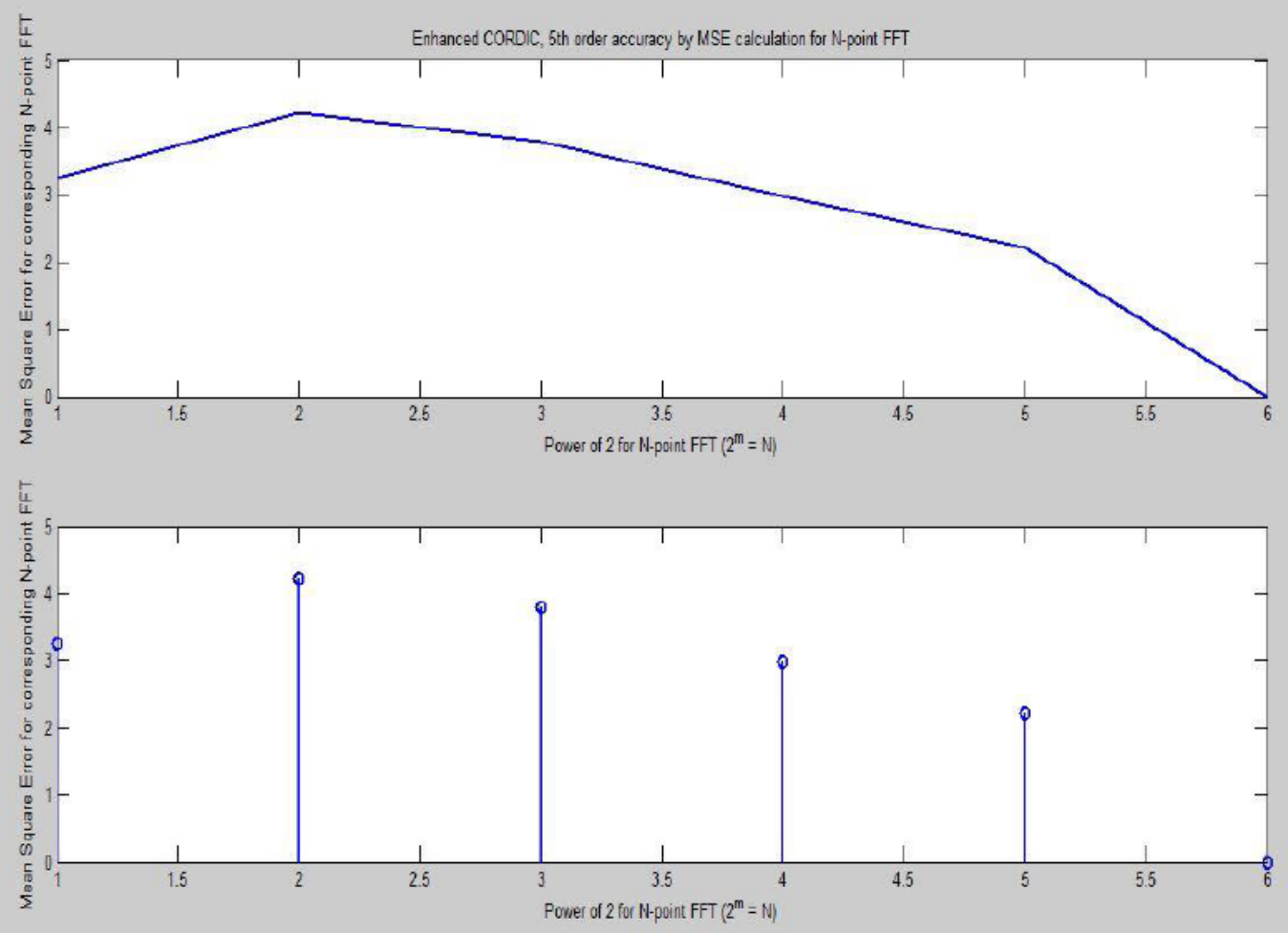

Figure 3 Enhanced CORDIC: FFT point till 64-point MSE result ( $5^{\text {th }}$ order accuracy)

Figure 1 shows that the mean square error reduces if the power of the transform is increased and it requires only adders and shifters to implement this as the scaling factor goes off in enhanced CORDIC compared to Simple CORDIC. 
Figure 2 also clarifies the same with $5^{\text {th }}$ order accuracy. Hence the as the order increases so does the accuracy.

From the above table and graph, we can see that by increasing the order there is a possibility of increase in accuracy but at the other end, the number of multipliers, adders and shifters also increase (increase in computational complexity). Hence, one need to balance this trade-off as per his requirement.

Table 2

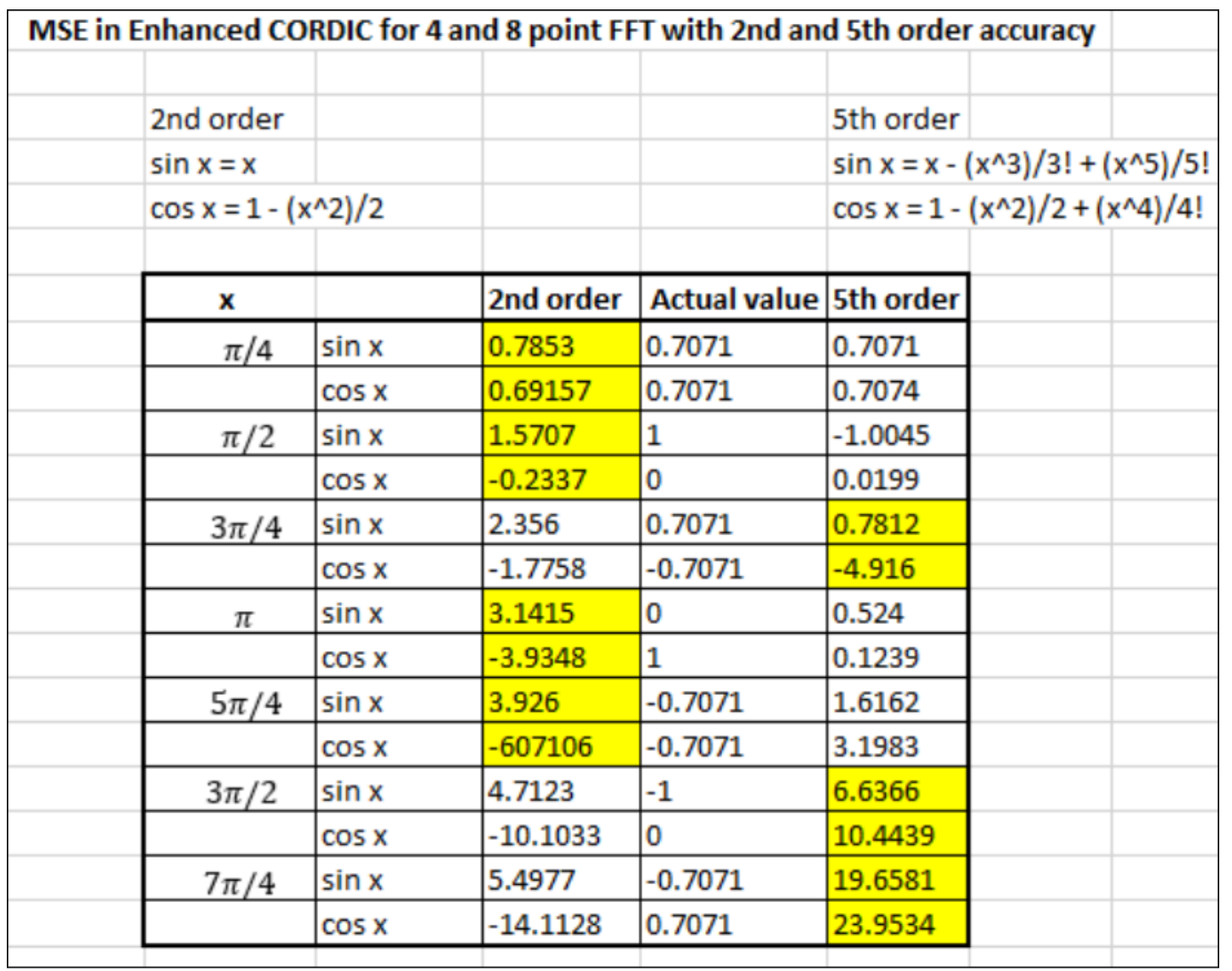

*Highlighted region show more error for that particular $\mathrm{x}$, compared to the values of other order

\subsection{Hyperbolic Trajectory using Extended Enhanced CORDIC}

The ROC in case of hyperbolic trajectory is 60.4 degrees. The results in the figure 3 to 8 shows various $\mathrm{N}$ points FFT coefficients calculated on the basis of Extended Enhanced CORDIC( $5^{\text {th }}$ order $)$. 

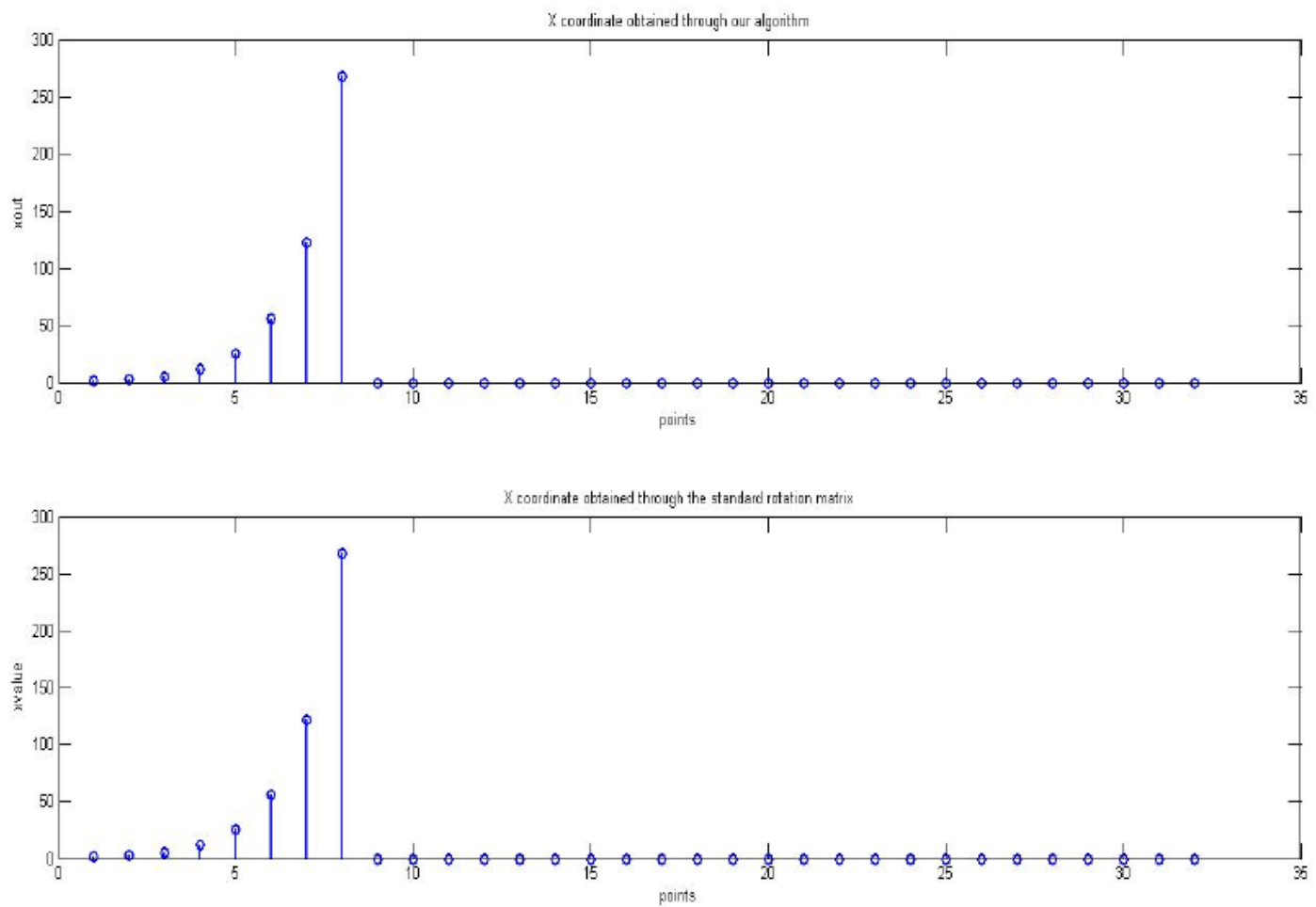

Figure $4 \mathrm{x}$-coordinates for 8 points in hyperbolic trajectory
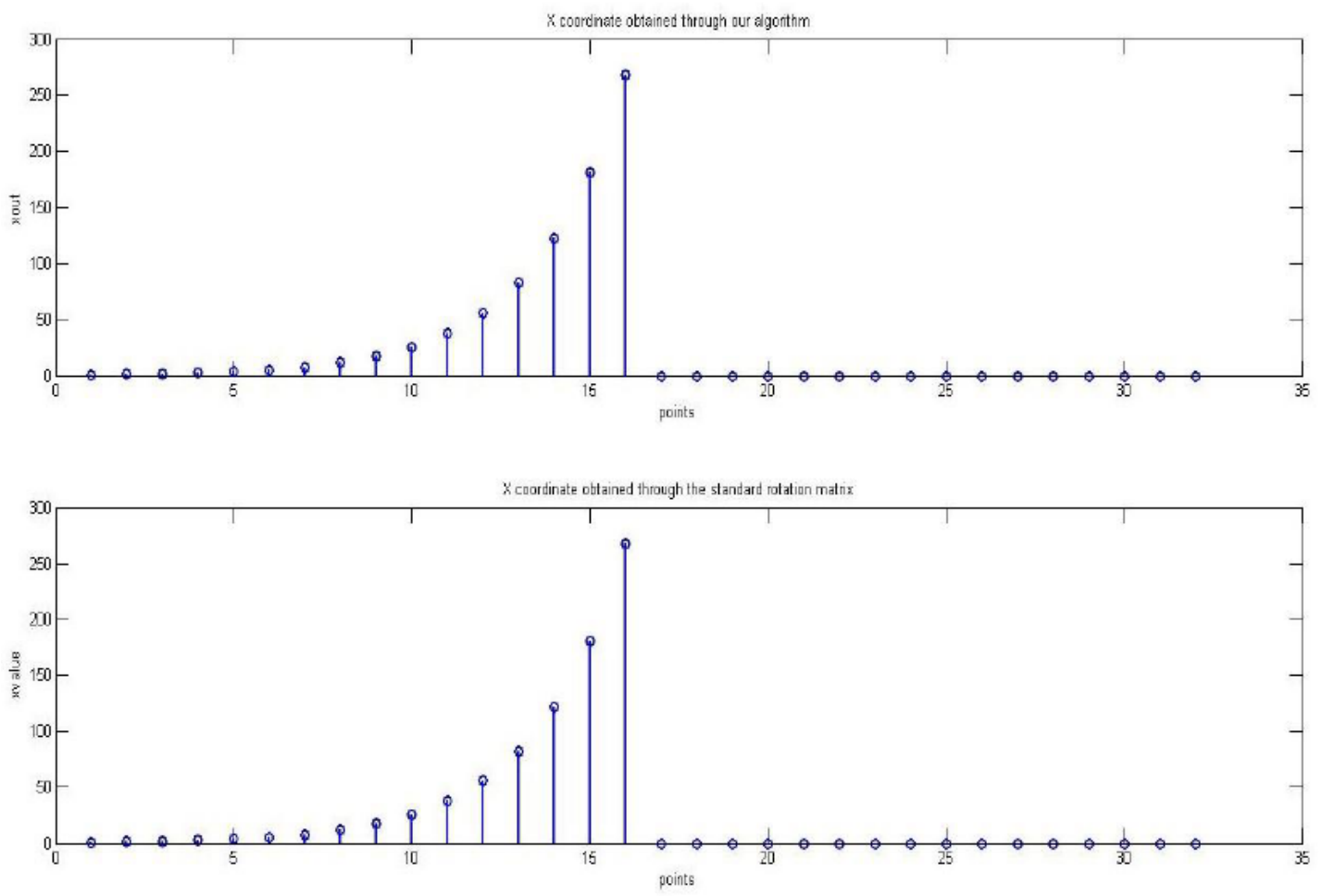

Figure $5 \mathrm{x}$-coordinates for 16 points in hyperbolic trajectory 
Improved Multiple Trajectories Rotator Design for Transforms
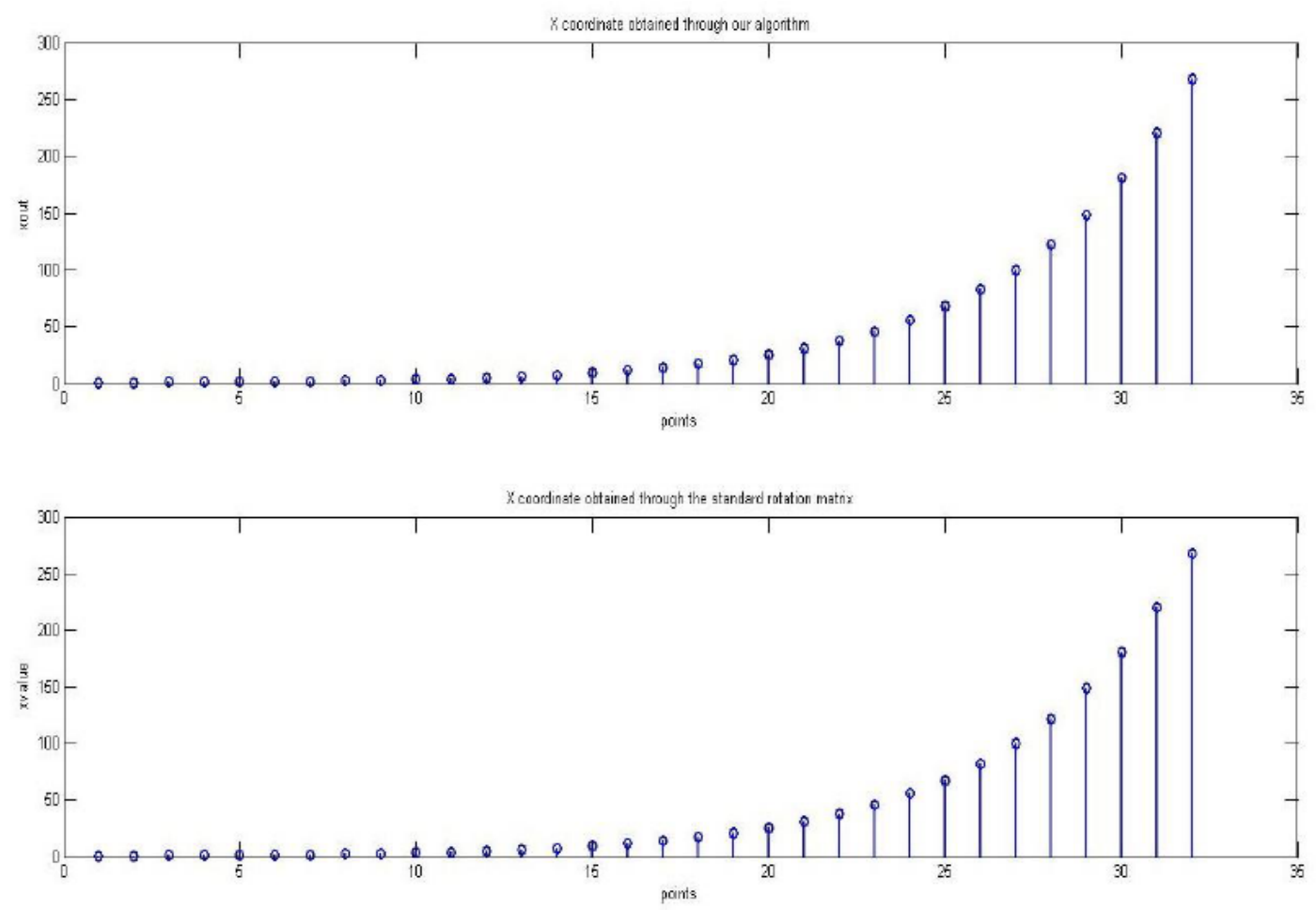

Figure $6 \mathrm{x}$-coordinates for 32 points in hyperbolic trajectory
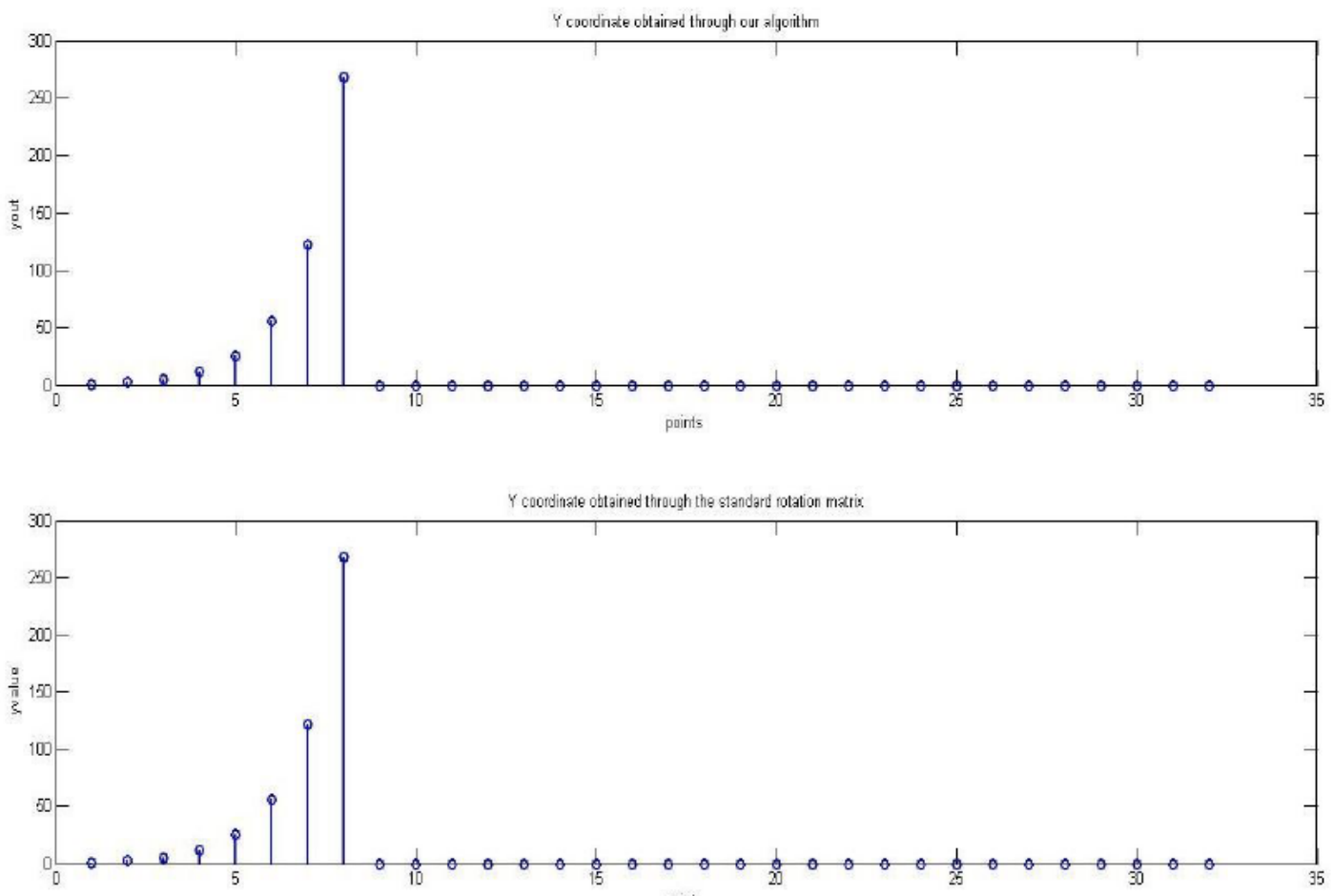

Figure 7. y-coordinates for 8 points in hyperbolic trajectory 

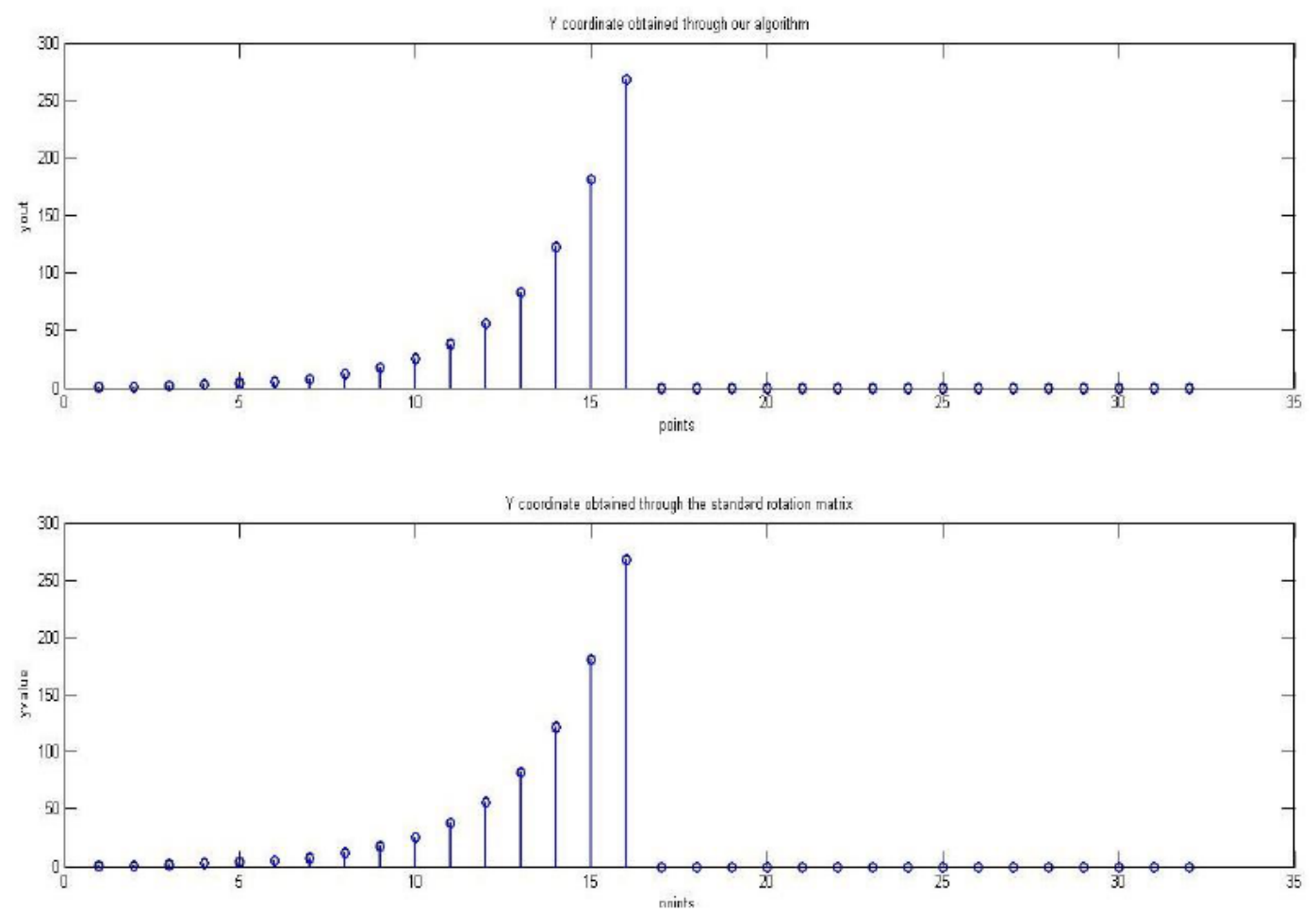

Figure 8 y-coordinates for 16 points in hyperbolic trajectory
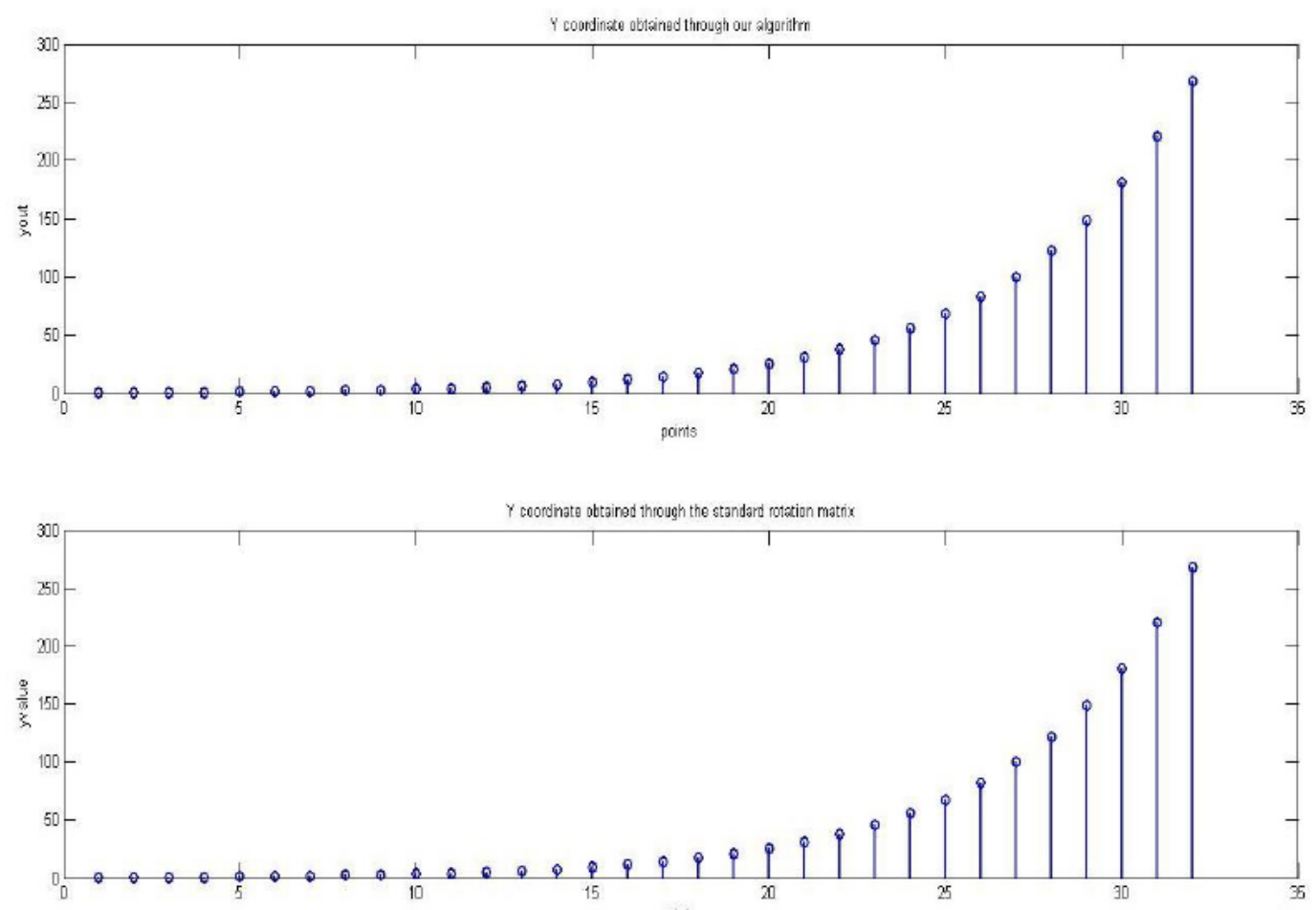

Figure 9 y-coordinates for 32 points in hyperbolic trajectory

\section{CONCLUSION}

We obtained the ROC and mean squared error of simple CORDIC for circular trajectory and for hyperbolic trajectory. We also obtained the ROC of enhanced CORDIC for circular trajectory. The errors through implementation were significantly less and were matching the values obtained through the rotation matrix for all trajectories. We 
implemented a unified algorithm with multiple trajectories. Hence multiple functionalities can be implemented just be adding a single parameter and by going till $5^{\text {th }}$ order the accuracy can be closer to the actual value. The hardware used for this will be only adders and shifters to implement the rotation and hence saving on the time as well.

\section{REFERENCES}

[1] A.G. Dempster, and M.D. Macleod, "Multiplication by two integers using the minimum number of adders," Proceedings - IEEE International Symposium on Circuits and Systems, pp. 1814-1818, 2005.

[2] Francisco J. Jaime, Miguel A. Sánchez, Javier Hormigo, Julio Villalba, and Emilio L. Zapata, "Enhanced Scaling-Free CORDIC," IEEE Transactions On Circuits And Systems-I: Regular Papers, VOL. 57, NO. 7, JULY 2010.

[3] Abhishek choubey, Mayuri Kulshreshtha, Karunesh, Determination of Optimum Fft for WiMax under Different Fading, International Journal of Electronics and Communication Engineering \& Technology (IJECET), Volume 3, Issue 1, January- June (2012), pp. 139-146

[4] Supriya Aggarwal, Pramod K. Meher, "Reconfigurable CORDIC Architectures for MultiMode and Multi-trajectory operations," IEEE International symposium on circuits and systems, June 2014.

[5] Dr. Syed Abdul Sattar, Dr. Mohammed Yousuf Khan and Shaik Qadeer, a New Radix-4 Fft Algorith, International Journal of Advanced Research in Engineering and Technology (IJARET), Volume 4, Issue 3, April 2013, pp. 251-256

[6] Supriya Aggarwal , Pramod Kumar Meher, Senior Member, IEEE, and Kavita Khare, "ScaleFree Hyperbolic CORDIC Processor and Its Application to Wave form Generation," IEEE Transactions On Circuits And Systems-I: Regular Papers,Vol.60,No.2, February 2013.

[7] Kamatham Harikrishna and T. Rama Rao, an Efficient Radix-22 Fft for Fixed \& Mobile Wimax Communication Systems, International Journal of Electronics and Communication Engineering \& Technology (IJECET), Volume 3, Issue 3, October- December (2012), pp. 265-279

[8] J.E. Volder, "The CORDIC Trigonometric Computing Technique," IRE Transactions on Electronic Computers, vol. EC-8, no.3, pp. 330-334.

[9] C. Basavaraju and Dr. Chandrakanth.H.G, Fft Based Spectrum Analysis Model for an Efficient Spectrum Sensing, International Journal of Advanced Research in Engineering and Technology (IJARET), Volume 5, Issue 12, December (2014), pp. 87-96

[10] Gurjit Singh, Rajeev Kumar, Dr. Manpreet Singh and Jujhar Singh Detection of Crack Initiation in the Ball Bearing Using FFT Analysis. International Journal of Mechanical Engineering and Technology, 8(7), 2017, pp. 1376-1382.

[11] J.S. Walther, "A Unified Algorithm for Elementary Functions," Conference Proceedings, Spring Joint Computer Conference, pp. 379-385, May 1971.

[12] Dr. Hanaa M and A. Salman, Information Hiding in Edge Location of Video Using Amalgamate $\mathrm{Fft}$ and Cubic Spline, International Journal of Computer Engineering and Technology (IJCET), Volume 4, Issue 4, July-August (2013), pp. 240-247

[13] N. Hariharavarshan, Jeyaram Durga Manian, and R. MelvinaMinny. Online Monitoring and Analysis of Induction Motor Using Current Signature Analysis Implementing Wavelet Analysis and FFT Analysis. International Journal of Electrical Engineering \& Technology, 7(6), 2016, pp. 36-54. 